\title{
L'émergence de la conscience francophone au Congo-Kinshasa
}

Jean-Christophe L. A. Kasende

\section{(2) OpenEdition \\ 12 Journals}

Édition électronique

URL : https://journals.openedition.org/dhfles/441

DOI : $10.4000 /$ dhfles.441

ISSN : 2221-4038

Éditeur

Société Internationale pour l'Histoire du Français Langue Étrangère ou Seconde

Édition imprimée

Date de publication : 1 janvier 2008

Pagination : 211-229

ISSN : 0992-7654

Référence électronique

Jean-Christophe L. A. Kasende, «L'émergence de la conscience francophone au Congo-Kinshasa »,

Documents pour l'histoire du français langue étrangère ou seconde [En ligne], 40/41 | 2008, mis en ligne le 18 décembre 2010, consulté le 27 mai 2021. URL : http://journals.openedition.org/dhfles/441 ; DOI :

https://doi.org/10.4000/dhfles.441

Ce document a été généré automatiquement le 27 mai 2021.

(C) SIHFLES 


\title{
L'émergence de la conscience francophone au Congo-Kinshasa
}

\author{
Jean-Christophe L. A. Kasende
}

\section{Le statut actuel du Congo-Kinshasa dans la francophonie internationale}

1 Dans la hiérarchisation qu'ils établissent, les auteurs du Dictionnaire général de la francophonie parlent de différents niveaux de la francophonie. Ce sont « d'abord les pays de langue française (France, Wallonie, Suisse romande, Québec...), puis les États qui ont choisi le français comme langue nationale à côté des idiomes autochtones, on dit alors que ce sont des contrées d'expression française (Liban, Sénégal, Zaïre $\left.{ }^{1} . ..\right)$, enfin les nations dont une minorité plus ou moins importante se sert du français comme instrument d'échange et de culture (Maghreb, Égypte...) ${ }^{2}$ ». À la dernière page du dictionnaire, les auteurs présentent les données statistiques et les informations concernant le Congo-Zaïre de la période allant de 1960-1965 puis de 1965-1970³ :

Le Zaïre (ex-Congo belge), avec $2344932 \mathrm{~km}^{2}$ et plus de 28 millions d'habitants, est par son étendue et après la France, le plus grand pays francophone du monde. Défini dans ses frontières par Léopold II, l'État indépendant du Congo (1885-1908) devenait le 18 octobre 1908 le Congo belge et l'un des pays les plus développés d'Afrique sur le plan économique. L'enseignement était donné d'abord dans la langue maternelle puis dans une des quatre langues nationales : kikongo (province de Kinshasa), tshiluba (Kasai), lingala (Équateur), kiswahili (Province orientale, Kivu, Shaba). Seule une élite de quelques milliers accédait à l'enseignement de l'une des deux langues nationales belges (français et néerlandais). L'enseignement en français fait des progrès sensibles depuis l'indépendance et la vie universitaire est active dans les trois campus de Kinshasa, Lubumbashi et Kisangani. On compte plus de 5 millions d'enfants scolarisés dans les classes primaires, 68000 élèves dans le secondaire et 3500 étudiants dans l'enseignement supérieur.

Il faut noter tout de suite que ces informations déjà archivées en 1977 par R. Cornevin dans son ouvrage intitulé Le Zaïre ont été simplement transcrites par les auteurs du Dictionnaire général de la francophonie en 1986, date de la publication de ce dernier; et 
qu'aujourd'hui, la situation de l'ancien Zaïre, redevenu la République démocratique du Congo, a considérablement évolué en termes de politique linguistique, de nombre d'habitants et donc de population scolarisée. Au point que le pays s'est hissé, en quarante-sept ans, depuis son accession à l'indépendance, au sommet de la majorité des pays africains comme la plus grande pépinière d'intellectuels francophones, pourvoyeuse d'enseignants de langue, culture et littérature francophones dans les universités américaines et européennes. Cette mise au point faite, il importe dans cette étude, de remonter dans le temps pour suivre le chemin, à la fois sinueux et hasardeux, qui a mené le Congo-Kinshasa à son statut et à sa place au sein de la famille francophone depuis son accession à l'indépendance le 30 juin 1960. L'adoption du français en tant qu'unique langue officielle du pays est l'aboutissement d'un long processus. Celui-ci fut basé sur l'émergence d'une certaine conscience historique de la francophonie chez les Congolais des deux générations qui se sont suivies, celle d'avant et celle d'après l'indépendance du pays. Le récit de ce processus, fondé en partie sur le souvenir capital de l'expérience familiale à un moment de notre enfance, est sans aucun doute celui que portent en eux la plupart des Congolais de la génération dite de la veille de l'indépendance. Il retrace le cheminement du Congo-Kinshasa, ancienne colonie belge, vers son accession à la francophonie, c'est-à-dire à son statut postcolonial d'un État souverain, membre du groupe d'États francophones ; c'est le récit et l'analyse des circonstances particulières qui ont conduit ce pays, non colonisé par la France, à son statut de deuxième pays francophone du monde en importance après l'Hexagone.

Le refus de l'administration coloniale belge d'encourager et de généraliser l'enseignement $d u$ français, l'image de Léopold Sédar Senghor, le discours de Brazzaville du général De Gaulle conférant à la France une nouvelle image, la naissance du mouvement indépendantiste au Congo-Kinshasa, l'influence des écoles locales de formation de l'élite catholique congolaise (notamment les petits séminaires) sont autant de points qui s'inscrivent dans le processus de «L'émergence du domaine et du monde francophones " au Congo-Kinshasa dans son ensemble; ils s'analysent comme les constituants d'une seule et unique causalité historique dans l'accession de ce géant de l'Afrique centrale à la francophonie internationale.

\section{L'aura senghorienne et l'imaginaire congolais des années 1960}

4 C'est dans une des rares occasions de réunion familiale, au cours d'une brève séance d'explications d'une collection de photos rassemblées par mon père dans son album que, pour la première fois, j'ai vu Léopold Sédar Senghor. Sa photo trônait à la première page de l'album à côté de celles de Patrice-Émery Lumumba, Kwame Nkrumah et Ahmed Sékou Touré, respectivement Premier ministre du Congo-Kinshasa, président du Ghana et président de la Guinée Conakry. Longtemps après, lorsque j'ai eu l'occasion de revoir la même image de Senghor, sur des couvertures d'ouvrages et des pages d'anthologies, avant de suivre au hasard des circonstances quelques-unes de ses interventions d'archives ou en direct à la télévision, j'avais déjà été marqué, voire conditionné, dès mon enfance, par le premier geste familial, par le discours admiratif de mon père pour l'homme: Senghor parlait français comme un Français, il avait enseigné le français aux Français. Sous son image, à la première page de l'album de mon 
père, ce dernier avait écrit en trois lignes : «Léopold Sédar Senghor, Président-poète du Sénégal, premier Noir professeur de français en France. »

5 La séance familiale d'album photos a eu lieu au camp des déplacés de guerre où nous étions, vers les années 1965, date de la fin officielle de la rébellion qui avait ensanglanté le Congo-Kinshasa, après l'assassinat en 1961 de Patrice-Émery Lumumba, le premier Premier ministre démocratiquement élu, après l'accession du pays à l'indépendance. Je devais reprendre mes études primaires interrompues à peine commencées deux ans plus tôt à cause de la guerre. Je comprenais déjà tous les mots que mon père avait employés pour présenter Senghor sur la première page de son album, sauf un seul: poète. Je lui demandai ce que le mot "poète " signifiait, et mon père me répondit en comparant le poète à notre tante surnommée Meka, griotte traditionnelle de la famille et berceuse occasionnelle d'enfants. Et mon père prit soin d'ajouter qu'à la différence de notre tante Meka, qui, elle, chantait en otetela, qui est notre langue maternelle, Senghor, lui, composait ses chansons (il voulait dire ses poèmes) en français, sans être français lui-même. Et, s'adressant à nous trois, mes deux frères aînés et moi, il ajouta encore ceci : «- Moi votre père, j'aurais été comme Senghor si j'avais eu la chance d'aller en France. » Puis détachant son regard de l'album qu'il feuilletait pour nous, il nous posa la question suivante : «- Qui parmi vous va être comme Senghor?» Pendant que mes frères, encore hésitants, se regardaient en souriant sans rien dire, je répondis sans réfléchir, presque instantanément, à la question: " - C'est moi.» Une réponse donnée spontanément, peut-être aussi bien pour avoir un rêve d'enfant à entretenir que pour obéir, je crois, a posteriori, au désir inassouvi de mon père, etc.

6 Je fais grâce au lecteur de la suite de cette causerie familiale entre mon père, mes frères et moi, pour renouer avec le sujet du colloque proprement dit. Je voudrais cependant indiquer que cette séquence de mon récit sert de témoignage à l'appui de « l'émergence de la conscience francophone au Congo-Kinshasa ». En effet, au Congo belge de la veille de l'indépendance, l'expression de la conscience sociale et politique, et donc francophone, a été assumée par les "évolués ». Ces derniers étaient une minorité d'indigènes sélectionnés et formés par le régime colonial belge pour remplir dans l'administration publique des fonctions auxiliaires (de commis, assistant médical ou agricole, etc.) ; ils constituaient la première génération d'intellectuels du futur Congo indépendant. Assistant médical diplômé, puis gérant et agent itinérant chargé d'inventaire et de contrôle de ventes d'une compagnie commerciale, mon père faisait partie de cette minorité privilégiée. C'est donc à ce titre que son récit spontané adressé à ses enfants tient lieu de témoignage authentique à l'appui de l'émergence de la conscience francophone au Congo-Kinshasa. En effet, dans la conscience collective de l'élite congolaise des années 1960, Senghor occupait une place de choix; le succès de son odyssée européenne, raconté aux générations montantes, était désigné à l'admiration de la jeunesse comme référence. Senghor était donc le modèle d'intellectuel africain à suivre, et pour tout dire, une séduction intellectuelle. Adolescent, puis jeune assistant d'université, je traînais, inconsciemment, comme beaucoup d'autres jeunes de ma génération, mon vœu secret d'enfant fait à ma famille et à la société de ressembler à Senghor. Il était pour moi une légende vivante, avant de se découvrir plus tard sous ses autres facettes de théoricien (parfois controversé) de la négritude, de mythe de l'Afrique et du monde, mais aussi d'artisan et fondateur du mouvement de la francophonie internationale aux côtés du général De Gaulle, président de la République française, Habib Bourguiba, premier président de la Tunisie 
indépendante et Hamani Diori, premier président de la République du Niger indépendant. Senghor souligne l'importance de sa rencontre avec le président tunisien, lorsqu'il rappelle l'origine du projet de la francophonie dans son discours de réception à l'Académie des sciences d'outre-mer le 2 octobre 1981 en ces termes :

L'idée m'en est venue, je crois, en 1955, lorsque, secrétaire d'État à la présidence du Conseil dans le Gouvernement Edgar Faure, j'étais chargé de la révision du titre VIII de la Constitution, relatif aux départements, territoires d'outre-mer et protectorats. J'eus, alors, l'occasion de m'en entretenir avec Habib Bourguiba, qui était en résidence surveillée en France. C'est de là que datent notre amitié et notre coopération. Il s'agissait, comme je l'ai dit en son temps, d'élaborer, puis d'édifier un Commonwealth à la française [...]. Par «ensemble ", j'entendais, j'entends toujours : entre nations qui emploient le français comme langue nationale, langue officielle ou langue de culture ${ }^{4}$.

7 J'ai compris longtemps après que, pour mon père qui appartenait à la première génération des quelques rares enfants indigènes scolarisés en langues vernaculaires sous le joug colonial belge, la maîtrise du français par un Africain colonisé était une fascination et devait tenir presque de la magie noire. Au Congo belge de l'époque en effet, la permission officielle d'enseigner le français aux indigènes colonisés ne fut donnée par les autorités coloniales belges que quelques années avant l'accession du Congo, alors colonie belge, à l'indépendance. Et cette langue qu'on leur interdisait d'apprendre au Congo, sauf si l'on choisissait de devenir prêtre, cette langue qu'il apprenait parfois en cachette parce que, son père ayant été polygame, il ne pouvait même pas mentir comme un de ses camarades sur sa vocation sacerdotale pour aller la maitriser au petit séminaire, - quitte à défroquer avant d'être ordonné prêtre -, cette langue du maître que tout semblait l'avoir empêché d'acquérir, cette langue de prestige, cette langue des Français, Senghor, un jeune homme noir du Sénégal, l'avait maîtrisée au point d'avoir pu l'enseigner aux jeunes Français de France. On comprend dès lors, en se fondant sur les faits historiques énumérés à l'instant, qu'à cette époquelà, un Congolais du Congo belge, comme mon père, ait pu trouver enviable la situation d'un Léopold Sédar Senghor. On peut extrapoler et dire qu'à l'échelle du pays déjà en proie à la fièvre de l'indépendance, cette analyse de mon père était partagée par tous les "évolués ", classe sociale à laquelle il appartenait. On peut donc affirmer sans aucune exagération, que c'est dans ce contexte que furent jetées les bases de l'émergence d'une conscience francophone au Congo-Kinshasa. Cette émergence d'une conscience francophone sera d'autant plus forte que l'opposition de l'administration coloniale à favoriser l'enseignement du français (à développer l'enseignement tout court) était, sinon explicitement radicale, du moins entretenue. Cette dernière affirmation sert de transition au point suivant portant sur la politique de l'administration coloniale en matière d'enseignement au Congo belge.

\section{L'administration coloniale et l'enseignement au Congo- Kinshasa}

8 Le paternalisme sévère, caractéristique du système colonial belge, est une des meilleures illustrations de la relation, au plan social et psychologique, entre le maitre et le subalterne. La position de supériorité absolue du maître colonisateur par rapport à celle d'infériorité toute aussi absolue du subalterne colonisé dans la hiérarchie établie par le système colonial ne pouvait, à la longue, que susciter chez les indigènes colonisés 
le désir ardent de ressembler au colonisateur. Dans tous les rapports de domination entre les peuples, cet état de chose est le fondement de la prise de conscience par les dominés du caractère inacceptable de leur situation qu'ils finissent par trouver inégalitaire et injuste.

Or, jusqu'aux années 1920, l'enseignement dispensé aux indigènes de la colonie au Congo belge était synonyme de la mission civilisatrice par la christianisation. En 1922, (alors qu'en Afrique Equatoriale Française on avait déjà célébré deux ans plus tôt la publication de Batouala, premier roman écrit en français par René Maran, fonctionnaire noir du territoire d'outre-mer), l'élaboration du système scolaire au Congo belge en était encore à ses premiers balbutiements. En 1922 donc, la commission constituée par Louis Franck, ministre de la colonie, précise les contours de l'enseignement au Congo belge : "La primauté devait être donnée à l'éducation plutôt qu'à l'instruction; les programmes et les méthodes devaient se préoccuper d'être adaptés au milieu 'indigène'. Par le fait même, l'enseignement devait se faire dans les langues locales. A l'époque, suivant l'opinion coloniale, la connaissance du français devait être évitée autant que possible, car elle était source d'orgueil et de prétention, disait-on ${ }^{5}$ ». Le destin anecdotique du célèbre écrivain congolais du Congo-Brazzaville, Sony Labou Tansi, est une illustration supplémentaire de la conscience collective des habitants des deux Congos voisins, conscience selon laquelle la seule connaissance de la langue de Voltaire était une garantie de l'instruction ou du savoir tout court. En effet, Sony Labou Tansi est né d'un père Congolais du Congo belge et d'une mère Congolaise du Congo français, tous deux de l'ethnie $(\mathrm{mu})$ kongo répartie sur les deux rives du fleuve Congo. Racontant au cours d'un entretien accordé à Bernard Magnier comment lui, enfant né sur les deux rives, était devenu du jour au lendemain habitant d'une seule rive, Sony explique la décision de son oncle maternel de lui faire traverser le fleuve au seul motif qu'au Congo belge sa scolarisation d'enfant indigène s'effectuait dans sa langue maternelle, le kikongo. L'écrivain rapporte les propos de son oncle à travers lesquels on sent l'inquiétude de ce dernier pour l'avenir de son neveu scolarisé en kikongo au Congo belge: "Mon oncle disait qu'il ne fallait pas aller à l'école pour ne rien apprendre et qu'il valait mieux dans ces conditions que je vienne au Congo français...6 ${ }^{6}$. Alors que le monde était en train de changer, le régime colonial belge au CongoLéopoldville en était encore, "pour rationaliser l'exploitation » à "former des agents indigènes, organiser des embryons d'administration et privilégier le colonisateur en l'auréolant de valeurs morales et culturelles prestigieuses ${ }^{7}$ ». Jean-Paul Sartre parle, en théorisant cette stratégie du système colonial, du souci de diviser pour régner; cela explique selon lui le fait que la métropole « a fabriqué de toutes pièces une bourgeoisie de colonisés ${ }^{8} »$. Cet obscurantisme du système colonial belge vis-à-vis des Congolais était entretenu au point qu'il s'est perpétué durant toute la période d'avant 1945 , comme l'explique encore Ndaywel dans son Histoire du Zaïre déjà citée :

La société belge avait ses problèmes et ses contradictions internes. Mais les Congolais en étaient si peu conscients, qu'ils continuaient à penser, du moins pour la plupart, que les Belges "étaient tous frères ». S'ils n'étaient pas ignorants de l'existence d'une langue flamande distincte du français, ils supposaient que cette seconde langue constituait le dialecte des Belges, de tous les Belges, et qu'ils en faisaient usage lorsqu'ils ne voulaient pas que les évolués comprennent le sens de leurs paroles. On supposait aussi, pour n'avoir pas eu la preuve du contraire, que tous les Blancs étaient des chrétiens, des collaborateurs des missionnaires. Les colons, les fonctionnaires coloniaux et les missionnaires s'étaient arrangés pour mettre cette solidarité apparente en avant. (p. 496) 
10 Pour confirmer la singularité de cette expérience belge au Congo-Kinshasa, on peut signaler qu'elle est aujourd'hui revendiquée comme un atout pour certaines recherches financées par l'Organisation internationale de la francophonie (OIF). Ainsi, pour préparer des dictionnaires africains plurilingues, les initiateurs du projet intitulé Réseau international des langues africaines et créoles (Rilac) ${ }^{9}$, revisitent en quelque sorte l'histoire de la francophonie, en exploitant à bon escient cet aspect de la réalité historique du Congo-Kinshasa. Pour fonder leur recherche sur les principes de la pédagogie convergente «qui consiste à fonder l'enseignement du français sur la connaissance des langues locales ", ils ont fait appel à Ngalasso Mwatha Musanji, un sociolinguiste du Congo-Kinshasa. L'équipe organisatrice de ce chantier affirme : «Il n'est pas étonnant que ce projet révolutionnaire ait été confié à un originaire de l'exCongo belge. Là-bas, le colonisateur faisait appel aux langues locales pour alphabétiser et commencer à scolariser les enfants africains. "

11 Mais toutes ces précautions prises par l'administration coloniale belge n'ont malheureusement pas produit les résultats escomptés. En effet, en interdisant l'enseignement du français au Congo pour l'isoler du reste du monde, l'administration coloniale belge a favorisé l'éveil de la conscience francophone chez les Congolais du Congo-Kinshasa ; elle a jeté ces derniers en quelque sorte dans les bras du général De Gaulle, ouvrant ainsi grandement à son ancienne colonie les portes de la grande famille francophonie. Mais il faut remonter aux années 1947, pour situer en quelque sorte l'origine de la pression exercée par les évolués sur le pouvoir colonial afin d'obtenir « la mise en place par les missions d'un réseau d'établissements d'enseignement secondaire général, distincts des petits séminaires ${ }^{10} »$. Malgré la création de ces premiers collèges d'enseignement secondaire général pour enfants autochtones en 1947 et la perspective de la sortie des premiers diplômés en 1953, l'université Lovanium (diminutif de Louvain) n'a pu ouvrir ses portes qu'en 1954. Par ailleurs, il faut souligner que dans l'énumération des événements qui furent à l'origine de l'éveil de la conscience de l'élite congolaise, l'historiographie du Congo-Kinshasa retient le discours de Brazzaville du général De Gaulle comme un fait important. Chronologiquement en effet, ce discours prononcé le 30 janvier 1944 est antérieur à la mobilisation des évolués en 1947 pour forcer le pouvoir colonial à fonder les collèges d'enseignement secondaire général qui poussèrent dans la foulée l'administration coloniale à accepter l'ouverture de l'université Lovanium par la suite. Quel est donc l'impact réel du discours du général De Gaulle sur l'éveil de la conscience francophone au Congo-Kinshasa ? C'est l'objet du point suivant de notre intervention.

\section{De Gaulle et l'éveil d'une conscience francophone au Congo-Kinshasa}

12 Après la Deuxième Guerre mondiale et l'occupation allemande, la France s'est vue menacée dans son influence en tant que puissance au sein de l'OTAN ; elle s'est sentie aussi ébranlée dans son hégémonie politique à travers le monde, après la perte de ses colonies et territoires d'outre-mer. Elle n'avait plus qu'une seule option pour garder sa place au sein de la communauté internationale témoin de son déclin en tant que puissance coloniale : profiter de l'avantage que lui donnait son histoire récente en tant qu'ancienne grande puissance coloniale dans le monde, pour maintenir sa place de puissance rivale face au bloc anglo-saxon. De Gaulle a vite compris l'importance de 
l'enjeu; il s'est employé à la construction d'un espace culturel, grâce à la langue française déjà implantée dans les territoires anciennement colonisés et devenue naturellement la langue principale d'expression commune. Son discours de Brazzaville entre autres, prononcé en janvier 1944, confirme aujourd'hui son statut de visionnaire. C'est donc sa capacité d'anticiper les événements et la venue des temps nouveaux qui lui a permis de réaliser son dessein, celui de se servir du français comme d'un outil fédérateur. Convaincu de l'avantage que lui accordait l'Histoire et «s'efforçant de redresser la France, il veut par tous les moyens pacifiques stimuler l'influence française dans le monde ${ }^{11} »$. Fin orateur, il a mis en pratique le principe rhétorique selon lequel « Il faut, [...] pour argumenter, attacher du prix à l'adhésion de son interlocuteur, à son consentement, à son concours mental ${ }^{12}$."

Le discours du général De Gaulle fut prononcé pour l'ouverture de la "Conférence de Brazzaville » sur l'union des territoires français d'outre-mer. Il n'était donc pas destiné aux Congolais du Congo belge. Mais l'élite congolaise du Congo-Kinshasa, qui était déjà frustrée par le paternalisme du système colonial belge, ne pouvait pas ne pas être curieuse de savoir ce qui se passait en dehors de son pays. La proximité des deux Congos, dont les capitales, Léopoldville et Brazzaville, ne sont séparées que par le fleuve Congo, favorisait la communication entre les deux peuples frères. Très vite, les échos de l'allocution du général De Gaulle aux « accents indépendantistes parvinrent à Léopoldville ». L'élite congolaise a dû être à la fois fascinée et séduite par la vision novatrice et l'attitude avant-gardiste du général De Gaulle lorsque ce dernier affirmait en janvier 1944 sa conviction en ces termes :

Mais, en Afrique française, comme dans tous les autres territoires où des hommes vivent sous notre drapeau, il n'y aurait aucun progrès qui soit un progrès, si les hommes, sur leur terre natale, n'en profitaient pas moralement et matériellement, s'ils ne pouvaient s'élever peu à peu jusqu'au niveau où ils seront capables de participer chez eux à la gestion de leurs propres affaires. C'est le devoir de la France de faire en sorte qu'il en soit ainsi ${ }^{13}$.

Bien que ce discours fût destiné aux ressortissants des territoires français d'Afrique, l'évolution rapide de la situation politique du côté français a eu un impact important sur l'attitude de l'élite du Congo belge. À côté de la France dont l'image devenait chaque jour fascinante et positive après cette intervention de Charles De Gaulle, la Belgique, c'est-à-dire la colonisation belge vécue au Congo-Kinshasa, est apparue aux yeux des Congolais comme un régime on ne peut plus inhumain. Alors que la France s'efforçait du mieux qu'elle pouvait de redorer son blason grâce au rôle historique et à l'activisme d'un général De Gaulle instruit pas les leçons de l'Histoire (il faut songer ici à l'occupation allemande suivie quelques années plus tard de deux défaites en 16 ans, dans la guerre d'Indochine et celle d'Algérie qui ont duré chacune 8 ans), la Belgique s'évertuait à agir comme si la Deuxième Guerre mondiale n'avait pas eu lieu. Dans son discours, De Gaulle, plus que d'autres dirigeants des puissances coloniales alliées (notamment la Grande-Bretagne et la Belgique) reconnaît la nécessité de tirer les leçons des quatre années de guerre et donc de l'occupation :

S'il est une puissance impériale que les événements conduisent à s'inspirer de leurs leçons et à choisir noblement, libéralement, la route des temps nouveaux où elle entend diriger les soixante millions d'hommes qui se trouvent associés au sort de ses quarante-deux millions d'enfants, cette puissance c'est la France ${ }^{14}$.

15 Même si le général De Gaulle agissait d'abord pour l'intérêt de la France, dans l'imaginaire de l'élite congolaise encore colonisée, la générosité et l'humanisme de la 
France contrastaient avec l'utilitarisme calculateur de la Belgique. En proclamant sa volonté d'ouvrir les territoires français de l'Afrique au reste du monde et en soulignant l'importance de la collaboration, au plus fort de la guerre, de l'Afrique Équatoriale et du Cameroun français avec les territoires voisins dont le Congo belge, De Gaulle associait à son insu l'élite congolaise du Congo-Kinshasa au projet de la francophonie. Plus tard, c'est grâce à la rencontre du général De Gaulle avec les dirigeants politiques des pays qui avaient entretenu des liens avec la France que le mot "francophonie " (avec sa forme adjectivale "francophone») va être utilisé dans son sens presque actuel, d'une réalité sociopolitique, d'une conscience francophone. Il sera popularisé dans ce deuxième sens, notamment par le président sénégalais, L. S. Senghor. Ce dernier «mérite une mention spéciale, pour avoir utilisé, le premier, le mot, après Onésime Reclus (1837-1916), dans un numéro spécial d'Esprit (novembre 1962) ${ }^{15} \%$. La francophoniesignifiait donc au départ dans l'esprit de ses promoteurs poussés par le souci de créer " une communauté d'intérêts ", « la conscience d'avoir en commun une langue et une culture francophones ».

En analysant aujourd'hui la structure oratoire du discours de Brazzaville du général De Gaulle, on comprend l'importance du rôle qu'il a joué dans le processus d'élaboration d'une communauté francophone. Pour atteindre son objectif, l'orateur qui avait en tête le projet de la création d'un Commonwealth à la française, a laissé à ses alliés africains, (notamment Senghor, Bourguiba et Diori) le soin de s'engager ouvertement dans la poursuite de cet aspect rationnel compromettant de son combat. Sachant qu'il avait le lourd héritage de l'empire colonial à justifier en partie, et surtout avec lequel il avait décidé de rompre, l'orateur a focalisé son attention sur les arguments contextuels, c'est-à-dire affectifs de son discours. Celui-ci était essentiellement fondé sur l'ethos et le pathos. Dans le premier cas (en parlant de l'ethos), Charles De Gaulle devait capter l'attention et gagner la confiance des Africains. Tout en essayant de souligner les bienfaits relatifs de l'empire colonial et l'importance de son œuvre, il promet de rompre avec un passé révolu pour répondre aux attentes de changement et d'amélioration des conditions de vie des habitants des territoires d'outre-mer. C'est cet aspect de la fonction oratoire, fondé sur l'ethos, que l'on peut attribuer à l'extrait suivant du discours :

Depuis un demi-siècle, à l'appel d'une vocation civilisatrice vieille de beaucoup de centaines d'années, sous l'impulsion des gouvernements de la République [...] les Français ont pénétré, pacifié, ouvert au monde, une grande partie de cette Afrique noire, que son étendue, les rigueurs du climat, la puissance des obstacles naturels, la misère et la diversité de ses populations avaient maintenue, depuis l'aurore de l'Histoire, douloureuse et imperméable. Ce qui a été fait par nous pour le développement des richesses et pour le bien des hommes, à mesure de cette marche en avant, il n'est, pour le discerner, que de parcourir nos territoires et, pour le reconnaître, que d'avoir du cœur. Mais, de même qu'un rocher lancé sur la pente roule plus vite à chaque instant, ainsi l'œuvre que nous avons entreprise ici nous impose sans cesse de plus larges tâches. Au moment où commençait la présente guerre mondiale, apparaissait déjà la nécessité d'établir sur des bases nouvelles les conditions de la mise en valeur de notre Afrique, du progrès humain de ses habitants et de l'exercice de la souveraineté française ${ }^{16}$.

En ce qui concerne le second cas (relatif au pathos), l'orateur devait exploiter des tendances, passions, désirs et émotions de son public africain pour obtenir son adhésion au projet qu'il avait à défendre. L'exploitation de cet aspect se traduit par la dénonciation dans le discours, en faveur des Africains des territoires français d'outre- 
mer, de l'inégalité et de l'injustice dont ils ont été victimes sous l'empire colonial ; par l'évocation de l'expérience douloureuse de la guerre partagée par la France et ses territoires d'outre-mer ; par l'expression de la reconnaissance de la France à l'égard des Africains pour leur participation aux efforts de la guerre; bref, par la prise en compte par la France du désir d'autodétermination des peuples africains des territoire d'outremer. Rappelons par ailleurs, qu'en soutenant contre certains parlementaires français, que la proposition de la loi sur l'auto-détermination des territoires de l'Afrique Occidentale Française présentée par L. S. Senghor soit soumise au référendum, le général De Gaulle a fait de ce dernier son fidèle allié, et à travers lui, Bourguiba et Diori.

18 Il n'était donc pas surprenant que l'aspect rationnel, purement syllogistique, de l'argumentation à l'appui de l'union des territoires français d'outre-mer ait été dévolu, comme nous l'avons affirmé plus haut, à ces trois alliés africains du général De Gaulle. L'argument peut se résumer en un enthymème à partir de l'analogie qu'établissent les alliés africains du général De Gaulle entre la Francophonie, version achevée de l'Union des territoires français d'outre-mer et le Commonwealth: l'Angleterre et ses anciennes colonies retrouvent l'unité dans le Commonwealth, la France dans la Francophonie. Mais ce qui importe ici, c'est l'analyse de la structure rhétorique du discours du général De Gaulle, pour souligner son impact sur l'adhésion des Africains des territoires français d'outre-mer en général et des Congolais du Congo-Kinshasa en particulier. Le discours de Brazzaville du général De Gaulle a donc balisé la voie qui a conduit à l'élaboration d'un projet francophone à l'échelle internationale. La structure oratoire du discours a parfaitement fonctionné dans un contexte où le statut de son auteur, représentant de l'empire colonial français, était de nature à susciter la méfiance des Africains.

19 Vue sous la double aura gaulliste et senghorienne, la France des années 1950 exerçait une véritable fascination sur l'élite congolaise du Congo-Kinshasa, suffisamment attentive en son temps à l'évolution du monde. À la différence de la Belgique, la France n'avait pas interdit dans ses colonies l'enseignement de sa langue aux enfants indigènes. Senghor, professeur de français en France, est donc l'incarnation de cette " générosité française » en matière de libéralisation de l'instruction et de la culture, si l'on peut s'exprimer ainsi, selon l'esprit de l'époque. Il faut reconnaître par ailleurs que, sur cet aspect précis de l'acquisition de la langue du colonisateur par le colonisé, la politique d'assimilation culturelle pratiquée par la France dans ses colonies était jugée positivement à l'époque par l'élite congolaise. Celle-ci s'estimait défavorisée comparativement aux ressortissants des colonies françaises de l'Afrique et d'ailleurs.

\section{En guise de conclusion : la pratique du français au Congo-Kinshasa}

20 On comprend dès lors qu'à la lumière de tout ce qui précède, le relent nationaliste récurrent, qui a toujours ponctué les relations belgo-congolaises souvent tumultueuses, n'a jamais affecté la politique linguistique congolaise en faveur du français. Celui-ci est d'ailleurs considéré avant tout comme la langue de la France et non celle de la Belgique, même si le français est l'une des deux langues officielles du royaume. En effet, si le français est aujourd'hui la langue officielle de la RDC grâce à la colonisation belge, cette dernière est plutôt accusée par le récit de la première génération de l'élite congolaise de n'avoir facilité ni son acquisition ni sa maîtrise par les indigènes. Cette conscience collective du refus de la Belgique d'ouvrir sa colonie au monde extérieur justifie, entre 
autres, l'adoption par les Congolais du français de France (plus précisément de Paris) et non celui de Belgique. C'est donc par dépit, je crois, qu'au Congo on dit soixante-dix et quatre-vingt-dix au lieu de septante et nonante comme en Belgique. Par ailleurs, toutes ces contingences historiques ont coïncidé avec un besoin local de cohésion nationale dans un pays considéré, à l'instar de la plupart des pays africains, mais lui plus que d'autres à cause de sa dimension géographique considérable (quatre ou cinq fois la France, et quatre-vingts fois la Belgique), comme une mosaïque de cultures exprimées par autant de langues différentes. Le français s'est donc imposé au Congo-Kinshasa comme une langue de cohésion nationale : il est la langue officielle, mais aussi celle de l'enseignement et de l'administration. Caractérisée par un plurilinguisme originellement dysfonctionnel à l'échelle nationale, parce qu'elle compte des centaines de langues, la RDC est donc le lieu par excellence de l'usage du français comme dénominateur national commun.

Et même dans la foulée de ce que l'on a appelé, sous le régime dictatorial de Mobutu, la politique de recours à l'authenticité zaïroise, la pertinence de l'utilisation du français comme langue officielle, langue de l'administration et langue de l'enseignement n'a pas été remise en question. Enfin, sous la même impulsion du nationalisme caractéristique de l'idéologie de l'authenticité, les participants au colloque national des linguistes zaïrois, tenu à Lubumbashi en 1974 sur la promotion des langues nationales, ne sont pas parvenus à dégager un consensus autour d'une seule langue susceptible de remplacer le français parmi les quatre langues nationales retenues, à savoir le tshiluba, le lingala, le kiswahili et le kikongo. Devenu francophone par un pur accident historique, le CongoKinshasa le demeure encore par nécessité. Mais pour combien de temps encore? En effet, les guerres de prédation rendant le gouvernement incapable de subvenir aux besoins de l'enseignement, l'activisme du bloc anglo-saxon et la quasi indifférence des pays francophones à la situation de crise chronique qui ronge ce pays font que l'on est aujourd'hui à mille lieues de l'enthousiasme que suscitèrent le discours du général De Gaulle le 30 juin 1944 et l'aura de L. S. Senghor. Qui va donc sauver ce géant de la francophonie qu'est le Congo-Kinshasa? Sans céder au pessimisme délirant de certains, je donne tout de même raison à Jean-Marc Léger qui met en garde la communauté francophone en comptant sur " la francophonie de la dernière chance ». "Chaque jour qui passe, dit-il, rend plus nécessaire cette entreprise [francophone], mais chaque jour la rend aussi plus incertaine et plus difficile. Dès lors, rien n'autorise encore de tenir pour acquis l'avènement d'une véritable communauté de langue française, ni $a$ fortiori à prédire la forme qu'elle revêtira, les voies qu'elle empruntera ${ }^{17}$. »

\section{BIBLIOGRAPHIE}

FANON, Frantz (2002). Les damnés de la terre, Paris, La Découverte \& Syros.

KADIMA-NZUJI, Mukala (1973). Bibliographie littéraire de la République du Zaïre (1931-1972), Lubumbashi, CELRIA, UnaZa. 
KOM, Ambroise (2000). Francophonie et dialogue des cultures. Mélanges offerts à Fernando Lambert, Université Laval (Québec), Crelca.

KOM, Ambroise (2000). « Francophonie et métissage culturel ; le senghorisme n'est-il qu'un anachronisme? ", in Ambroise Kom, Francophonie et dialogue des cultures. Mélanges offerts à Fernando Lambert, Université Laval (Québec), Crelca, 2000, p. 37-49.

LABOU TANSI, Sony (1986). « Entretien avec Bernard Magnier », in Équateur, n 1, Paris.

LÉGER, Jean-Marc (1987). « La francophonie de la dernière chance », in Jean-Marc Léger, La francophonie : grand dessein, grande ambiguïté, Québec, Hurtubise, p. 179-192.

LÉGER, Jean-Marc (1987). La francophonie : grand dessein, grande ambiguité, Québec, Hurtubise. LUTHI, Jean-Jacques, VIATTE Auguste et ZANANIRI Gaston (1986). Dictionnaire général de la francophonie, (Haut Comité de la Francophonie et du Secrétariat Général de la Langue Française), Paris, Letouzey et Ané.

MAUGEY, Axel (2001). De la francophonie québécoise à la francophonie internationale, Québec, Humanitas.

NDAYWEL è NZIEM,Isidore (1997). L'histoire du Zaïre. De l'héritage ancien à l'âge contemporain, Louvain-La-Neuve, Duculot.

PERELMAN, Chaïm et OLBRECHTS-TYTECA, Lucie (1983). Traité de l'argumentation. La nouvelle rhétorique, Bruxelles, Édition de l'université de Bruxelles.

RUBANGO, Nyunda ya (2001). Les pratiques discursives du Congo-Belge au Congo-Kinshasa, Paris, L'Harmattan.

SARTRE, Jean-Paul (2002). «Préface à l'édition de 1961 », Frantz Fanon, Les damnés de la terre, Paris, La Découverte \& Syros, 2002, p. 17-36.

<http://www.rinoceros.org/spip.php?auteur183\&lang=fr>

(page consultée en septembre 2007)

<http://www.charles-de-gaulle.org/article.php3?id_article=52\&page=1>

(page consultée en septembre 2007)

$<$ http://www.ladocumentationfrancaise.fr/dossiers/francophonie/fondateurs.shtml> (page consultée en septembre 2007)

\section{NOTES}

1. L'actuelle République démocratique du Congo (RDC), appelée également Congo-Kinshasa, a été désignée par les dénominations suivantes: Congo belge ou Congo-Léopoldville, pendant la période coloniale ; la République du Zaïre ou le Zaïre tout court, sous la dictature de Mobutu.

2. Jean-Jacques Luthi, Auguste Viatte et Gaston Zananiri, "Préface »,Diction-naire général de la francophonie, p. 1.

3. R. Cornevin intitulé Le Zaïre, 1977, et Mukala Kadima-Nzuji,Bibliographie littéraire de la République du Zaïre (1931-1972), (sources citées par Jean-Jacques Luthi, Auguste Viatte et Gaston Zananiri, auteurs du Dictionnaire général de la francophonie).

4. <http://www.ladocumentationfrancaise.fr/dossiers/francophonie/fondateurs.shtml>

5. Isidore Ndaywel è Nziem, L'histoire du Zaïre. De l'héritage ancien à l'âge contemporain, p. 399.

6. Sony Labou Tansi, «Entretien avec Bernard Magnier », in Équateur, nº 1, Paris, 1986, p. 10. 
7. Ambroise Kom, «Francophonie et métissage culturel; le senghorisme n'est-il qu'un anachronisme? », in Ambroise Kom (dir.), Francophonie et dialogue des cultures. Mélanges offerts à Fernando Lambert, p. 39.

8. Jean-Paul Sartre, « Préface à l'édition de 1961 », Frantz Fanon, Les damnés de la terre, p. 20.

9. <http://www.rinoceros.org/spip.php?auteur183\&lang=fr>, page consultée le 27 septembre 2007.

10. Isidore Ndaywel è Nziem, op. cit., p. 502.

11. Axel Maugey, De la francophonie québécoise à la francophonie inter-nationale, p. 16.

12. Ch. Perelman, L. Olbrechts-Tyteca, Traité de l'argumentation. La nouvelle rhétorique, p. 20-21.

13. <http://www.charles-de-gaulle.org/article.php3?id_article=52\&page=1>

14. Ibid.

15. Nyunda ya Rubango, Les pratiques discursives du Congo-Belge au Congo-Kinshasa, p. 227.

16. Op. cit.

17. Jean-Marc Léger, "La francophonie de la dernière chance», inJean-Marc Léger, La francophonie : grand dessein, grande ambiguité, p. 179-192.

\section{RÉSUMÉS}

La place de la RDC dans la francophonie internationale aujourd'hui est l'abou-tissement d'un processus historique aléatoire allant de retentissants échos, à Léopoldville, du discours de Brazzaville du général De Gaulle, à l'imaginaire congolais nourri par l'aura senghorienne des années 60 , en passant par l'attitude contreproductive du système colonial belge.

Le refus de l'administration coloniale belge de généraliser l'enseignement du français au Congo contraste, en la matière, avec « l'humanisme » et " la générosité culturelle » du système colonial français. La France a en effet encouragé le développement de l'enseignement dans ses territoires d'outre-mer jusqu'à faire de Senghor le premier Africain noir professeur de français en France. La prise de conscience par l'élite congolaise de son retard par rapport à ses homologues des colonies françaises est le fondement de «l'émergence du domaine et du monde francophones » en RDC. Ainsi donc, bien que le Congo-Léopoldville soit devenu francophone grâce à la colonisation belge, la Belgique est plutôt accusée de n'avoir facilité ni l'acquisition du français ni sa maîtrise par les autochtones.

Cette conscience collective du refus de la Belgique d'ouvrir sa colonie au monde extérieur justifie, semble-t-il, l'adoption par les Congolais de la RDC du français parisien. En effet, au CongoKinshasa on dit "soixante-dix » et "quatre-vingt-dix » au lieu de "septante » et "nonante » comme en Belgique. Il faut reconnaître enfin qu'outre tous ces aléas historiques, avec ses quelques centaines des langues ethniques, toutes fonctionnelles, la RDC, aujourd'hui premier pays francophone du monde en nombre d'habitants, semblait avoir son destin profondément enraciné dans la francophonie: langue officielle, mais aussi langue de l'enseignement et de l'administration publique, le français y est surtout la langue de cohésion nationale.

The status of DRC in the international French-speaking world today is the culmination of an unpredictable historical process based on the three following important factors: the resounding echoes in Leopoldville of General De Gaulle's opening speech of Brazzaville Conference in 1944, the Congolese imagination enriched by the senghorian aura of the sixties, and the counterproductive attitude of the Belgian colonial system. 
The refusal of the Belgian colonial administration to teach French to the Congolese contrasted with the French colonial system founded the cultural assimilation. It is because of this French colonial system that Senghor became the first black African teacher of French in France. The awareness of its delay in comparison with the natives of the French colonies helped "the emergence of French-speaking conscience" among the Congolese elite. Therefore, although Congo-Léopoldville became a French-speaking country through colonization, the Belgian colonial system did nothing to help Congolese study French.

The public awareness of the colonial system's refusal to open its colony to the world justifies the choice made by the Congolese people to use Parisian French spoken by the educated people of Paris and not Belgian French. In Congo-Kinshasa, indeed, people say "soixante-dix" and "quatrevingt-dix" instead of "septante" and nonante". Finally it is necessary to acknowledge that besides all these historical vagaries, with its ethnic and very functional languages, the DRC is today the number one French-speaking country in the world in terms of the number of inhabitants. Its destiny appears deeply rooted in the French-speaking world. Besides being the language of both education and public administration, French, as the sole official language, also insures the national cohesion.

\section{INDEX}

Mots-clés : conscience francophone, francophonie, Congo-Kinshasa ou Congo belge, français langue officielle et langue de l'enseignement et de l'administration

Keywords : French-speaking conscience, french-speaking country, Congo-Kinshasa or Belgian Congo, French as the sole official language, the language of education and public administration

\section{AUTEUR}

JEAN-CHRISTOPHE L. A. KASENDE

Département d'études françaises

Université Dalhousie

Nouvelle-Écosse, Halifax, Canada 